\title{
MINIMAL IDEALS IN NEAR-RINGS AND LOCALIZED DISTRIBUTIVITY CONDITIONS
}

\author{
GARY BIRKENMEIER and HENRY HEATHERLY
}

(Received 30 May 1991)

Communicated by P. Schultz

\begin{abstract}
Let $B, S$, and $T$ be subsets of a (left) near-ring $R$ with $B$ and $T$ nonempty. We say $B$ is $(S, T)$-distributive if $s\left(b_{1}+b_{2}\right) t=s b_{1} t+s b_{2} t$, for each $s \in S, b_{1}, b_{2} \in B, t \in T$. Basic properties for this type of 'localized distributivity' condition are developed, examples are given, and applications are made in determining the structure of minimal ideals. Theorem. If $I$ is a minimal ideal of $R$ and $I^{k}$ is $\left(I^{m}, I^{n}\right)$-distributive for some $k, n \geq 1, m \geq 0$, then either $I^{2}=0$ or $I$ is a simple, nonnilpotent ring with every element of $I$ distributive in $R$. Theorem. Let $R^{k}$ be $\left(R^{m}, R^{n}\right)$-distributive, for some $k, n \geq 1, m \geq 0$; if $R$ is semiprime or is a subdirect product of simple near-rings, then $R$ is a ring. Connections are established with near-rings which satisfy a permutation identity and with weakly distributive near-rings. If $R \rightarrow A \rightarrow 0$ is an exact sequence of near-rings, then conditions on $A$ are given which will impose conditions on the minimal ideals of $R$.
\end{abstract}

1991 Mathematics subject classification (Amer. Math. Soc.): 16 A 76.

A minimal ideal of a ring (and hence the heart of a subdirectly irreducible ring) is either square zero or a simple ring [11, p. 135]. It is easy to find near-rings which have a minimal ideal which is neither square zero nor a simple near-ring. Zero symmetric examples are more difficult to locate, but Kaarli has exhibited one which is also finite and abelian [15]. The quest to characterize minimal ideals in near-rings has proved to be a difficult one, even in the zero symmetric and distributively generated cases. Significant results have been attained in this classification problem by Scott [19] and Kaarli $[14,16]$. This paper continues the investigation of the problem begun in $[3$,

(C) 1993 Australian Mathematical Society 0263-6115/93 \$A2.00+0.00 
4] and most recently carried out in [7]. Of equal importance herein is the introduction of localized distributivity conditions. We develop some basic properties of what we call ' $(S, T)$-distributive' (which is such a condition), and use this condition to investigate the structure of minimal ideals. This proves especially useful in conjunction with a general permutation identity condition, where a structure theorem for near-rings with descending chain conditions on ideals is given (Proposition 3.2). We also develop conditions under which a minimal ideal of a near-ring is a ring. (A tour through the zoo of standard near-ring examples reveals this often happens). Several examples are discussed in detail to illustrate that the localized distributivity conditions discussed do occur in some natural examples (for example, in certain nearrings of mappings). These examples illustrate and also show some of the limits to the theory developed.

Herein 'near-ring' will always mean 'left near-ring'. Except where noted, the notation and terminology is that in [17]. Let $A$ and $B$ be nonempty subsets of a near-ring $R$. Then $\langle A\rangle_{R}$ is the ideal in $R$ generated by $A$. We use $(0: A)_{R}=\{b \in R: A b=0\}$, the right annihilator of $A$ in $R$. Define $[A, B]=\{a b-b a: a \in A, b \in B\}$. We will be particularly interested in the ideal $\langle[A, B]\rangle_{R}$, which will be denoted by $\langle A, B\rangle_{R}$. We use $\mathscr{D}(R)$ for the set of all distributive elements in $R$. In all of the above notation, if no ambiguity will arise we suppress the subscript $R$. As is standard we use $A \cdot B=\{a b: a \in A, b \in B\}$. The additive subgroup of $R$ will be denoted by $R^{+}$or $(R,+)$ as seems convenient. We use $R^{(m)}$ for the $m$-th commutator subgroup of $R^{+}$and let $R^{(1)}=R^{\prime}$.

A near-ring $R$ is said to satisfy a permutation identity if there exists a non-identity permutation $\sigma$ on $n$ letters such that for each $a_{1}, \ldots, a_{n} \in R$, $\Pi a_{j}=\prod a_{\sigma(j)}, j=1, \ldots, n$. If $\sigma(n) \neq n$, we say " $R$ satisfies a permutation identity which moves the last place". Such permutation identities play an important role herein. (For more on rings or near-rings which satisfy a permutation identity see $[3,5,6]$, where inter alia, a substantial bibliography on the subject can be found.)

\section{Localized distributivity}

In this section $R$ will always denote a near-ring.

Definition 1.1. Let $B, S$, and $T$ be subsets of $R$ with $B$ and $T$ nonempty. We say $B$ is $(S, T)$-distributive if

$$
s\left(b_{1}+b_{2}\right) t=s b_{1} t+s b_{2} t
$$

for all $b_{1}, b_{2} \in B, s \in S$, and $t \in T$. (If $S$ is empty, delete the corresponding factor in the above equation). Note that if $T$ is the largest subset of $R$ 
for which $B$ is $(S, T)$-distributive and if $B T \subseteq B$, then $T$ is semigroup.

Observe that a distributive near-ring $D$ is $(\varnothing, D)$-distributive and in a pseudo-distributive near-ring $K$, see [13], $K^{2}$ is $(\varnothing, K)$-distributive. Any near-ring $R$ is $(\varnothing, \mathscr{D}(R))$-distributive. In the sequel we often make use of nonempty sets $T$ for which $T^{n}$ is $\left(T^{m}, T^{k}\right)$-distributive, where $k, m$, and $n$ will always be integers such that $m \geq 0, k, n \geq 1$. Here we define $T^{0}=\varnothing$. For example, if $R^{n} \subseteq \mathscr{D}(R)$ then $R$ is $\left(\varnothing, R^{n}\right)$-distributive; if $\left(R^{n}, \cdot\right)$ is a commutative semigroup, then $R$ is $\left(R^{n-1}, R^{n}\right)$-distributive. If $B$ is $(\varnothing, T)$-distributive, then $B$ is $(S, T)$-distributive for any $S \subseteq R$. Also, if $B^{k}$ is $\left(S^{n}, T\right)$-distributive and $S \subseteq B$, then $B$ is $\left(S^{n+k-1}, T\right)$ distributive.

LEMMA 1.2. Let $S$ be a subsemigroup of $(R, \cdot)$ and $B$ a nonempty subset of $R$ such that $S B \subseteq S$. If $S$ satisfies a permutation identity $\sigma$ of length $n$ with $\sigma(n)=k<n$, then:

(1) $S^{n}[B, B]=0$.

(2) If either $B$ or $S$ is a subsemigroup of $R^{+}$, then $B$ is $\left(S^{k}, S^{n-k}\right)$ distributive.

Proof. (1) This can be proved using the proof of the lemma in [19] with the obvious modifications.

(2) Let $s_{1}, s_{2}, \ldots, s_{n} \in S$ and $x, y \in B$. Consider

$$
\begin{aligned}
s_{1} s_{2} & \cdots s_{k-1}\left[s_{k}(x+y)\right] s_{k+1} \cdots s_{n}=s_{\sigma(1)} s_{\sigma(2)} \cdots s_{\sigma(n-1)}\left[s_{k}(x+y)\right] \\
& =s_{\sigma(1)} s_{\sigma(2)} \cdots s_{\sigma(n-1)}\left(s_{k} x\right)+s_{\sigma(1)} s_{\sigma(2)} \cdots s_{\sigma(n-1)}\left(s_{k} y\right) \\
& =\left(s_{1} \cdots s_{k} x s_{k+1} \cdots s_{n}\right)+\left(s_{1} \cdots s_{k} y s_{k+1} \cdots s_{n}\right) .
\end{aligned}
$$

Hence $B$ is $\left(S^{k}, S^{n-k}\right)$-distributive.

EXAMPLE 1.3. Let $R$ be a near-ring which satisfies a permutation identity $\sigma$ of length $n$, where $\sigma(n)=k<n$. By a proof similar to that of Lemma $1.2(2), R$ is $\left(R^{k-1}, R^{n-k}\right)$-distributive. In particular if $R$ is right permutable (that is, $a b c=a c b$ for $a, b, c \in R)$, then $R$ is $(R, R)$ distributive.

Many examples of right permutable near-rings which are not distributive are given in [2], including ones which are abelian. A method for constructing right permutable, distributively generated near-rings which are not distributive is given in [3, Example 4.9]. Note that if $R$ is a nilpotent near-ring of index $n$, then $R$ satisfies every permutation identity of length at least $n$; hence $R$ is $\left(R^{k-1}, R^{n-k}\right)$-distributive for all $1 \leq k \leq n$. 
In contrast, a left permutable near-ring $R$ (that is, $a b c=b a c$ for all $a, b, c \in R)$ need not be $\left(R^{m}, R^{n}\right)$-distributive for any $m, n$. For example, near-ring number 36 on $S_{3}$ in [10] is left permutable, distributively generated, and is not $\left(R^{m}, R^{n}\right)$-distributive for any allowable $m, n$.

There are many near-rings among those given via Cayley tables or Clay representations which are $(S, T)$-distributive, and for which $T$ is not made up entirely of distributive elements. But rather than display a plethora of these somewhat artificial examples, we feel the following very natural examples will suffice.

EXAMPLE 1.4. Let $H$ and $K$ be nonzero subgroups of a nonabelian group $(G,+)$, with $K \subseteq H$. Let $E(G, H)$ be the subgroup of $M_{0}(G)$ generated by the set of all homomorphisms from $G$ into $H, \operatorname{Hom}(G, H)$, and let $R$ be the subgroup of $M_{0}(G)$ generated by all the elements of $\operatorname{Hom}(G, H)$ which fix $K$ setwise. Observe that $E(G, H)$ and $R$ are distributively generated near-rings; and if $K$ is a fully invariant subgroup of $H$, then $R=E(G, H)$. Let $S=\{\sigma \in R: G \sigma \subseteq K\}$ and let $T$ be all elements of $S$ that are endomorphisms when restricted to $K$. Then $R$ is $(S, T)$-distributive and $S$ is a two-sided $R$-subgroup of $R$. If $K$ is abelian, then $S=T$ and $S$ is a ring. If $K$ is normal in $H$, then $S$ is an ideal of $R$. Thus if $K$ is a fully invariant, abelian subgroup of $H$, then $R=E(G, H), R$ is $(T, T)$-distributive, and $T$ is a ring and an ideal of $R$. Recall that if $H$ is solvable, then such a non-trivial, fully invariant, abelian subgroup of $H$ will exist.

We next give a special case of this construction.

EXAMPLE 1.5. Let $(B,+)$ be a solvable group of length $n+1$, with $n$ positive. Let $G=B^{(n)} \oplus B, H=\left\{(0, b): b^{\prime} \in B\right\}$, and $K=\left\{(0, c): c \in B^{(n)}\right\}$. Then $K$ is a fully invariant, abelian subgroup of $H$. Using the notation of Example 1.4 we see that $R=E(G, H)$ is a distributively generated nearring which is $(T, T)$-distributive, and $T$ is an ideal of $R$. In general, $T \nsubseteq \mathscr{D}(R)$, as can be seen from the specific example where $B=S_{3}, B^{\prime}=A_{3}$, and $G=B^{\prime} \oplus B$. Define $\psi_{1}$ and $\psi_{2}$ on $G$ as follows: $(a, b) \psi_{1}=(0, a)$ and $(a, b) \psi_{2}=(0, b)$. Then $\alpha, \psi_{2} \in R$ and $\beta, \psi_{1} \in T$, where $\alpha=\psi_{1}+\psi_{2}$ and $\beta=\psi_{1}+2 \psi_{2}$. Note that $\left(\alpha+\psi_{2}\right) \beta \neq \alpha \beta+\psi_{2} \beta, \beta^{3}=\beta$, and $\psi_{1}^{2}=0$. Hence $T$ contains a nonzero idempotent element, a nonzero nilpotent element, but $T \nsubseteq \mathscr{D}(R)$.

EXAMPLE 1.6. Let $A$ and $B$ be nonempty subsets of a near-ring $R$ such that $R=A+B=\{a+b: a \in A, b \in B\}, A^{m} B=0, A$ is $\left(A^{m}, A^{n}\right)$ distributive for some $n, m \geq 1$, and $R B \subseteq B$. Then $R$ is $\left(A^{m}, R^{n}\right)$ distributive. There are many examples of this where $R$ is both $(A, R)$ distributive and $(B, R)$-distributive, but $R$ is neither distributively generated nor is it $\left(R^{m}, R^{n}\right)$-distributive for any $m, n \geq 1$. 
Concrete examples of this phenomenon are near-rings number 24 and 27 on $S_{3}$ in [2], where $A=\{0, a\}$ and $B=\{0, x, y\}$. These near-rings are zero symmetric, and subdirectly irreducible with heart $B$.

A special case of Example 1.6 occurs where $A$ and $B$ are ideals of $R, R=$ $A \oplus B$, and $A$ is $(A, A)$-distributive, which yields $R$ is $(A, R)$-distributive.

The following lemmas are somewhat technical, but they lead to our main theorem of this section. This theorem is used repeatedly in the sequel to obtain various characterizations of minimal ideals.

LemMA 1.7. Let $B$ be $a(S, T)$-distributive subset of $R$ with $a, b \in B$, $s \in S$, and $x, y \in T$.

(1) If $T$ is closed under addition, then $s b x+s a y=s a y+s b x$.

(2) If $x \in T$ implies $x+x \in T$, then $s b x+s a x=s a x+s b x$.

(3) If $0 \in B$, then $0 T=0$. If also $0 T R=0$, then $R$ is zero symmetric.

(4) If 0 and $-B$ are in $B$, then $s(-b) x=-(s b x)$ and $s(a-b) x=$ $s a x-s b x$.

(5) If $B$ is a subgroup of $R^{+}$and $T$ satisfies the condition that $x \in T$ implies $x+x \in T$, then $S\left(B^{\prime}\right) T=0$.

(6) If $G$ is a subgroup of $R^{+}$and $B G \subseteq B$, then $s b\left(\sum g_{i}\right) x=\sum s b g_{i} x$, where $g_{i} \in G, i=1, \ldots, n$.

Proof. The following arguments will also work for $S=\varnothing$ by deleting the factor $s$.

(1) Consider $s(a+b)(x+y)=s(a+b) x+s(a+b) y=s a x+s b x+s a y+s b y$. Also $s(a+b)(x+y)=s a(x+y)+s b(x+y)=s a x+s a y+s b x+s b y$. By cancellation, $s b x+s a y=s a y+s b x$.

(2) Proceed as in part (1) with $x=y$.

(3) Observe $0 x=s(0+0) x=0 x+0 x$. Thus $0 x=0$.

(4) Consider $0=s 0 x=s(b-b) x=s(b+(-b)) x=s b x+s(-b) x$. Hence $s(-b) x=-(s b x)$. Consequently $s(a-b) x=s a x-s b x$.

(5) This follows from the previous parts.

(6) The proof is by induction on $n$. The key induction step is given by:

$$
\begin{aligned}
s b\left(g_{1}+\cdots+g_{n}\right) x & =s\left[b\left(g_{1}+\cdots+g_{n-1}\right)+b g_{n}\right] x \\
& =s b\left(g_{1}+\cdots+g_{n-1}\right) x+s b g_{n} x .
\end{aligned}
$$

LEMMA 1.8. Let $I$ be a two-sided R-subgroup such that $I^{k}$ is $\left(I^{m}, I^{n}\right)$ distributive, where $k, n \geq 1$ and $m \geq 0$. Then:

(1) $R$ is zero symmetric; 
(2) if $\varnothing \neq Y \subseteq R$ such that $I^{k+m} Y I^{n}=0$, then $I^{k+m}\langle Y\rangle_{R} I^{n}=0$.

Proof. (1) From Lemma $1.7(3), 0 I^{n}=0$ and in particular $0 \cdots 0 t=0$, for each $t \in I$; hence, $0 I=0$. Since $I^{n} R \subseteq I$, Lemma 1.7 (3) yields $R$ is zero symmetric.

(2) Let $a_{1}, a_{2}, a_{3} \in R, s \in I^{m}, v \in I^{k}, t \in I^{n}$, and $y \in Y$. First observe that

$$
0=s v\left[a_{1}+\left(-a_{1}\right)\right] t=s\left[v a_{1}+v\left(-a_{1}\right)\right] t=s v a_{1} t+s v\left(-a_{1}\right) t
$$

so $s v\left(-a_{1}\right) t=-\left(s v a_{1} t\right)$. Then using Lemma 1.7 (6), we have that each of the terms $s v y t, s v\left(a_{1}+y-a_{1}\right) t$, and $s v\left[\left(y+a_{2}\right) a_{3}-a_{2} a_{3}\right] t$ is zero. Consequently, $I^{k+m}\langle Y\rangle_{R} I^{n}=0$.

LEMMA 1.9. Let $B$ and $S$ be subsemigroups of $(R, \cdot)$ such that $S$ satisfies a permutation identity and $B$ is $\left(S^{m}, S^{n}\right)$-distributive for integers $m \geq 0$ and $n \geq 1$.

(1) If 0 and $-B$ are contained in $B$ and $B S$ and $S B$ are in $S$, then there exists a positive integer $h$ such that $S^{h}[B, B] S^{h}=0$.

(2) If $B=R$ and $S$ is a semigroup ideal of $(R, \cdot)$, then there exists $a$ positive integer $j$ such that $S^{j}\langle R, R\rangle S^{j}=0$, and $R$ is zero symmetric.

Proof. (1) There exists a smallest positive integer $q$ such that for all $u, v \in S^{q}$ and all $x, y \in S$, we have $u x y v=u y x v$ [18, Theorem 1]. Let $k=\max \{m, n, q\}$. Then $B$ is $\left(S^{k}, S^{k}\right)$-distributive. Let $s_{1}, s_{2}, s_{3}, s_{4} \in$ $S^{k}$ and $a, b \in B$. Consider

$$
s_{1}\left(s_{2} a\right)\left(b s_{3}\right) s_{4}=s_{1}\left(b s_{3}\right) s_{2}\left(a s_{4}\right)=s_{1}\left(s_{2} b\right) s_{3}\left(a s_{4}\right)=s_{1} s_{3}\left(s_{2} b a\right) s_{4}=s_{1} s_{2} b a s_{3} s_{4} \text {. }
$$

Let $h=2 k$. Using Lemma $1.7(4), S^{h}[B, B] S^{h}=0$.

(2) This follows from part (1) and a proof similar to that of Lemma 1.8.

The distributor set of $R$ is: $\Delta(R)=\{(a+b) c-b c-a c: a, b, c \in R\}$. The ideal $\langle\Delta(R)\rangle_{R}$ is called the distributor ideal of $R$. If no ambiguity will result we will write $\Delta$ for $\Delta(R)$ and $\langle\Delta\rangle$ for $\langle\Delta\rangle_{R}$. (The notation for the distributor set and the distributor ideal are not standard in the literature. The concept was introduced by Fröhlich [12] in the setting of distributively generated near-rings, and has been used in classifying near algebras by Brown $[8,9])$. Note that $R$ is distributive if and only if $\Delta(R)=0$. An ideal $I$ of $R$ contains $\Delta(R)$ if and only if $R / I$ is distributive; so $\langle\Delta(R)\rangle \subseteq\langle R, R\rangle$. If $R$ is distributively generated, then $R / R^{\prime}$ is a ring; so in this case $\langle\Delta(R)\rangle \subseteq R^{\prime}$. 
For near-rings in general this need not hold, even if $R^{\prime}=0$. The next lemma presents some useful, albeit obvious, connections between $\langle\Delta\rangle,\left\langle R^{\prime}\right\rangle$, and $\langle R, R\rangle$.

LEMMA 1.10. Let $R$ be a near-ring.

(1) $\langle\Delta\rangle \subseteq\langle R, R\rangle$.

(2) $0 \cdot R \subseteq\langle\Delta\rangle$.

(3) $\left\langle R^{\prime}\right\rangle^{2} \subseteq\langle\Delta\rangle$ and if $R^{2}=R$ then $\left\langle R^{\prime}\right\rangle \subseteq\langle\Delta\rangle$.

(4) If $R$ is distributively generated, then $\langle\Delta\rangle \subseteq\left\langle R^{\prime}\right\rangle=R^{\prime}$.

(5) If $R$ is distributively generated and $R=R^{2}$, then $\langle\Delta\rangle=R^{\prime}$.

THEOREM 1.11. Let I be a two-sided $R$-subgroup such that $I^{k}$ is $\left(I^{m}, I^{n}\right)$ distributive, where $k, n \geq 1$ and $m \geq 0, a, b \in R$ and $t, w \in I$.

(1) $I^{k+m}\left\langle R^{\prime}\right\rangle_{R} I^{n}=0=\left(I \cap\left\langle R^{\prime}\right\rangle_{R}\right)^{k+m+n+1}$.

(2) $I^{k+m}\langle\Delta\rangle_{R} I^{n}=0=\left(I \cap\langle\Delta\rangle_{R}\right)^{k+m+n+1}$.

(3) If I contains no nonzero nilpotent two-sided $R$-subgroups, then $I$ is $a$ ring and $I \subseteq \mathscr{D}(R)$. Furthermore $(t+w) b=t b+w b$, at $+b t=$ $b t+a t$, and $t a+t b=t b+t a$.

(4) If $I$ is also an ideal of $R$ and satisfies a permutation identity then $\langle I, R\rangle_{R}$ is nilpotent.

Proof. Let $a, b \in R, v \in I^{k}, s \in I^{m}, x \in I^{n}$, and $Y$ be a subgroup of $R$. Note that if $y=y_{1} y_{2} \cdots y_{j}$, where $y_{i} \in Y$ then $y+y=y_{1} y_{2} \cdots$ $y_{j-1}\left(y_{j}+y_{j}\right) \in Y^{j}$.

(1) Note $-I^{k} \subseteq I^{k}$. From Lemma 1.7 parts (2), (4) and (6) we have $s v(a+b-a-b) x=0$. Applying Lemma 1.8 yields the desired result.

(2) From Lemma $1.7(6), s v[(a+b) c-b c-a c] x=0$. Applying Lemma 1.8 yields the result.

(3) The conclusions of this part are immediate consequences of parts (1) and (2) and the assumption.

(4) Observe $\langle I, R\rangle_{R} \subseteq I$. Using the proof of Lemma 1.9 and letting $I=S, a \in I$, and $b \in R$ will yield this part.

Note that if $R$ satisfies a permutation identity given by a permutation $\sigma$ of length $n$ and $\sigma(n)=k<n$, then $R$ is $\left(R^{k-1}, R^{n-k}\right)$-distributive and $\left\langle R^{\prime}\right\rangle,\langle\Delta(R)\rangle$, and $\langle R, R\rangle$ are nilpotent.

For a ring $A$, if $I$ is an ideal of $A$ and $B$ an ideal of $I$, then $\langle B\rangle_{A}^{3} \subseteq B$, the Andrunakievic Lemma [11, p. 107] or [1]. This lemma has proved useful 
in the study of minimal ideals, subdirectly irreducible rings, and radicals for rings. An immediate consequence of this lemma is that a minimal ideal of $A$ is either a simple nonnilpotent ring or it is square zero. Like many such useful and elementary results in ring theory, the strict near-ring analogue of Andrunakievic's Lemma is false. An example given by Kaarli for other purposes shows this even in the case of a finite, abelian, zero symmetric near-ring [15, pp. 77-78]. For in this near-ring, called $N$, there is a unique minimal ideal $S$, which is not nilpotent, and the near-ring $S$ has a nonzero ideal $U$ which is square zero. Thus no power of $S$ can be contained in $U$.

We are able to preserve some essence of the Andrunakievic Lemma.

Proposition 1.12. Let $I$ be an ideal of a zero symmetric near-ring $R$ such that $I^{k}$ is $\left(I^{m}, I^{n}\right)$-distributive for some $k, n \geq 1, m \geq 0$. If $B$ is any nonzero ideal of the near-ring $I$, then $\left(\langle B\rangle_{R}\right)^{j} \subseteq I^{m} I^{k}\langle B\rangle_{R} I^{n} \subseteq B$, where $j=k+m+n+1$.

Proof. This follows from Lemma 1.7 (6) and a straightforward calculation.

Note in the above proposition if $I$ is a minimal ideal, then $I^{j} \subseteq B$.

\section{Minimal ideals}

In this section all near-rings are zero symmetric. We note some properties of minimal ideals in general and give sharper results where certain localized distributivity conditions hold. Necessary and sufficient conditions are given for a minimal ideal to be a ring.

Proposition 2.1. Let $I$ be a minimal ideal of a near-ring $R$ with $I^{2} \neq 0$. Then $(0: I)$ is a prime ideal of $R$ and either.

(1) $(0: I)=0$ and $R$ is subdirectly irreducible with heart I; or

(2) $\bar{R}=R /(0: I)$ is subdirectly irreducible with heart $\bar{I}$, the isomorphic image of $I$ under the natural homomorphism $R \rightarrow \bar{R}$.

Proof. First note that, by the assumption in this section, $R$ is zero symmetric, and so $(0: I)$ is an ideal. Observe that if $X$ is any ideal of $R$ such that $I \cap X=0$, then $X \subseteq(0: I)$. Consider ideals $A, B$ of $R$ such that $A B \subseteq(0: I)$. If neither $A$ nor $B$ is a subset of $(0: I)$, then $I \cap A$ and $I \cap B$ are each different from zero and hence $I$ is contained in $A$ and in $B$. So 
$I^{2} \subseteq A B \subseteq(0: I)$; this is a contradiction since $I^{2} \subseteq I$ and $I \cap(0: I)=0$. Thus $(0: I)$ is a prime ideal of $R$.

If $(0: I)=0$, then for each nonzero ideal $X$ of $R$ we have $I \cap X \neq 0$ and consequently $I \subseteq X$, yielding $R$ is subdirectly irreducible with heart $I$.

Next take $(0: I) \neq 0$. Suppose $\bar{I}$ contains a nonzero ideal of $\bar{R}$. Then this ideal has the form $\bar{T}$, where $T$ is an ideal of $R$ and $(0: I) \subseteq T$. Since $I \cap T=0$ implies $T \subseteq(0: I)$ and hence $\bar{T}$ is zero, we have $I \cap T \neq 0$, and consequently $\bar{I}=\bar{T}$; so $\bar{I}$ is a minimal ideal of $\bar{R}$. Then $I$ maps onto the minimal ideal $\bar{I}$ under the natural homomorphism $R \rightarrow \bar{R}$, and since $I \cap(0: I)=0$ we need $I$ and $\bar{I}$ are isomorphic near-rings. If $\bar{r}=r+(0: I)$ in $\bar{R}$ satisfies $\bar{I} \bar{r}=0$, then $\operatorname{Ir} \subseteq(0: I)$. But $I r \subseteq I$, so $I r=0$ and hence $\bar{r}=0$. Thus $\bar{R}$ is subdirectly irreducible with heart $\bar{I}$.

THEOREM 2.2. Let $I$ be a minimal ideal in a near-ring $R$. Then $I^{k}$ is $\left(I^{m}, I^{n}\right)$-distributive, for some $k, n \geq 1, m \geq 0$ if and only if either $I^{2}=0$ or $I$ is a simple nonnilpotent ring with $I \subseteq \mathscr{D}(R)$. In particular, if $R$ or $I$ is a distributive near-ring, then $I^{2}=0$ or $I$ is a nonnilpotent simple ring.

Proof. If $I$ is nilpotent of index $q \geq 2$, then $I=\left\langle I^{q-1}\right\rangle_{R}$. Write a general element $y \in I$ in the format of an element of $\left\langle I^{q-1}\right\rangle_{R}$. Then a routine calculation shows $x y=0$ for each $x \in I$, yielding $I^{2}=0$. Assume $I^{2} \neq 0$. By Theorem 1.11, $I \subseteq \mathscr{D}(R)$. Furtermore assume, without loss of generality (Proposition 2.1), that $R$ is subdirectly irreducible with $I$ as its heart. If either $R^{\prime}$ or $\Delta(R)$ is nonzero then, by Theorem $1.11, I$ is nilpotent. Hence $R$ is a ring. The converse is obvious.

COROLLARY 2.3. Let $I$ be a minimal ideal of a near-ring $R$, with $I^{2} \neq 0$. Then exactly one of the following holds:

(1) $I$ is a simple ring, $I \subseteq \mathscr{D}(R)$, and $R /(0: I)$ is a subdirectly irreducible prime ring;

(2) $I=\langle\Delta(I)\rangle_{R} \subseteq\langle\Delta(R)\rangle_{R}$ and $I^{+}$is solvable of length $n$, for some $n$;

(3) $I=\langle\Delta(I)\rangle_{R}=\left\langle I^{(n)}\right\rangle_{R} \subseteq\langle\Delta(R)\rangle_{R} \cap\left\langle R^{(n)}\right\rangle_{R}$, for each $n$.

Proof. If $\Delta(I)=0$, then $I$ is a distributive near-ring and Theorem 2.2 yields $I$ is a simple ring. This allows us to use Theorem $1.11(1)$ and (2) to get $I \cap\left\langle R^{\prime}\right\rangle$ and $I \cap\langle\Delta(R)\rangle$ are zero, and hence $\left\langle R^{\prime}\right\rangle$ and $\langle\Delta(R)\rangle$ are contained in $(0: I)$. This and Proposition 2.1 yield that $R /(0: I)$ is a subdirectly irreducible prime ring. So take $\Delta(I) \neq 0$. Then $I=\langle\Delta(I)\rangle_{R} \subseteq$ $\langle\Delta(R)\rangle_{R}$. If $I^{+}$is not solvable, then $I^{(n)} \neq 0$ for each $n$ and hence (3) follows immediately. 
If in the above corollary we assume $R$ is distributively generated, then case (2) cannot occur, for we have shown that in this situation $I^{+}$is either abelian (and hence $I$ is a simple ring) or $I^{+}$is a perfect group [7, Proposition 10]. If furthermore either $R^{\prime}$ or $\langle\Delta(R)\rangle$ are multiplicatively nilpotent, then only case (1) remains possible. This nilpotency of $R^{\prime}$ will occur if $R^{+}$is a solvable group [17, Corollary 9.49] or if $R$ is weakly distributive [17, Corollary 9.55]. We immediately have:

COROLlARY 2.4. Let $R$ be a subdirectly irreducible nonring. If either.

(1) $R$ is distributively generated and $R^{+}$is solvable, or

(2) $R$ is weakly distributive,

then the heart of $R$ is square zero.

Proposition 2.5. Let I be a minimal ideal in a near-ring $R$, with $I^{2} \neq 0$. If I satisfies a permutation identity $\sigma$ of length $j$ and if either:

(1) $I^{k}$ is $\left(I^{m}, I^{n}\right)$-distributive for some $k, n \geq 1, m \geq 0$; or

(2) $\sigma$ does not leave $j$ fixed;

then $I$ is a field, $R=I \oplus(0: I)$, and $I \subseteq \mathscr{D}(R)$.

Proof. Recall from Example 1.3 that (2) implies (1). By Theorem 2.2, I is a simple ring which satisfies a permutation identity. Such a ring is either a field or square zero [6]. Let $e$ be the identity element for the field $I$. Then using the Peirce decomposition for this idempotent yields: $R=(0: e)_{R}+e R$. Note that $(0: e)_{R}=(0: I)$ and $e R=I$ to obtain $R=(0: I) \oplus I=I \oplus(0: I)$.

This proposition extends a result of Wiegandt [20] in the case where $R$ is subdirectly irreducible. Furthermore, it answers, in the affirmative, the question raised in [3] as to whether a minimal ideal in a right permutable near-ring must be either nilpotent or a simple near-ring.

Proposition 2.6. Let $R \rightarrow B \rightarrow 0$ be an exact sequence of near-rings, where $R \rightarrow B$ has kernel $K$. If $B$ is distributively generated, then every minimal ideal of $R$ which is not contained in $K$ is either square zero or is a subdirectly irreducible near-ring.

Proof. Let $I$ be a minimal ideal of $R$ with $I \nsubseteq K$ and $I^{2} \neq 0$. Then $I \cap K=0$ and hence $K \subseteq(0: I)$. Observe that $B$ maps homomorphically onto $\bar{R}=R /(0: I)$ under the natural homomorphism induced on $B \approx R / K$ by the ideal $(0: I) / K$. So $\bar{R}$ is distributively generated. Using Proposition 2.1 we have $\bar{I} \approx I$ and $\bar{I}$ is a minimal ideal of $\bar{R}$. Kaarli [16] has shown 
that a minimal ideal in a distributively generated near-ring is either square zero or is a subdirectly irreducible near-ring.

Proposition 2.7. Let $R \rightarrow B \rightarrow 0$ be an exact sequence of near-rings, where $R \rightarrow B$ has kernel $K$. If $B$ has descending chain condition on right $B$-subgroups and is either distributively generated or has unity, then every minimal ideal of $R$ which is not contained in $K$ is either square zero or is a simple near-ring.

Proof. The proof is similar to that of Proposition 2.6, using a result due to Kaarli [14] which says that under the appropriate descending chain condition a near-ring which is either distributively generated or has unity has the property that every minimal ideal is square zero or is a simple near-ring.

Proposition 2.8. Let $R \rightarrow B \rightarrow 0$ be an exact sequence of near-rings, where $R \rightarrow B$ has kernel $K$. Assume $B$ satisfies a permutation identity $\sigma$ of length $j$. If either.

(1) $B^{k}$ is $\left(B^{m}, B^{n}\right)$-distributive for some $k, n \geq 1, m \geq 0$; or

(2) $\sigma$ does not leave $j$ fuxed;

then every minimal ideal of $R$ which is not contained in $K$ is either square zero or is a field.

Proof. Recall (2) implies (1). The proof is then similar to that of Proposition 2.6, using $\bar{R}^{k}$ is $\left(\bar{R}^{m}, \bar{R}^{n}\right)$-distributive and using Proposition 2.5.

Note that in Propositions 2.6, 2.7 and 2.8 if $B$ and $R \rightarrow B$ can be found so that $K$ is nilpotent, then the classification of minimal ideals in $R$ is substantially advanced.

\section{Miscellaneous applications}

In this section we make use of "localized distributivity" in some contexts other than that of minimal ideals and make use of some of the results of sections 1 and 2 . All near-rings in this section are zero symmetric.

Proposition 3.1. Let $R$ be a near-ring with $R^{k}$ being $\left(R^{m}, R^{n}\right)$-distributive for some $k, n \geq 1, m \geq 0$.

(1) If $R$ is simple, then either $R^{2}=0$ or $R$ is a ring. 
(2) If $R$ has no nilpotent, nonzero ideals, then $R$ is a semiprime ring. If also $R$ has descending chain condition on right (left) ideals, then $R$ is a finite direct sum of full matrix rings over skewfields.

(3) If $R$ is a subdirect product of simple near-rings, then $R$ is distributive, and the nonnilpotent subdirect product components are rings.

Proof. As an immediate consequence of Theorem 2.2, using $I=R$ we get (1). From Theorem 1.11, if $R$ has no nilpotent ideals, then $\Delta(R)=R^{\prime}=0$ and hence $R$ is a ring. Invoking the descending chain condition on right (left) ideals and using the Artin-Wedderburn Theorem gives the rest of (2). In part (3), Theorem 2.2 yields $R$ is distributive.

Observe that in (2) we have that $R$ will have no nonzero nilpotent right or left ideals; however there are zero symmetric near-rings with no nonzero nilpotent ideals which do have nonzero nilpotent left ideals [10, Number 10 on $K_{4}$ ].

Proposition 3.2. Let $R$ be a near-ring with descending chain condition on ideals and let $S=\operatorname{soc} R$. Assume $S$ satisfies a permutation identity $\sigma$ of length $j$. If either.

(i) $S^{k}$ is $\left(S^{m}, S^{n}\right)$-distributive for some $k, n \geq 1, m \geq 0$; or

(ii) $\sigma$ does not leave fixed;

then $R=A \oplus B$, where $R^{\prime} \cup \Delta(R) \cup[R, R] \subseteq A,(\operatorname{soc} A)^{2}=0, B$ is a finite direct sum of minimal ideals of $R$ each of which is a field, and $B \subseteq \mathscr{D}(R)$.

Proof. Use Proposition 2.5 repeatedly to obtain $R=A_{t} \oplus\left(I_{1} \oplus \cdots \oplus I_{t}\right)$, where $R^{\prime} \cap \Delta(R) \cup[R, R] \subseteq A_{t}$ and each $I_{i}$ is a minimal ideal of $R$ and is a field. The chain $A_{1} \supseteq A_{2} \supseteq \ldots$ must terminate, finitely, and hence the process stops, yielding the desired decomposition.

Note that if to the hypothesis of Proposition 3.2 we add the condition:

(iii) $S$ contains no nonzero nilpotent ideals;

then the conclusion becomes: $R$ is a finite direct sum of fields.

Proposition 3.3. Let $R$ be a near-ring with $R^{k}$ being $\left(R^{m}, R^{n}\right)$ distributive for some $k, n \geq 1, m \geq 0$. If $M$ is a maximal ideal of $R$, then either:

(1) $R^{2} \subseteq M$ and $M$ is maximal as a normal subgroup of $R^{+}$; or

(2) $\left\langle R^{\prime}\right\rangle \cup\langle\Delta(R)\rangle \subseteq M$ and $M$ is maximal as a two-sided $R$-subgroup. 
Proof. The homomorphic image $T=R / M$ has $T^{k}$ being $\left(T^{m}, T^{n}\right)$ distributive. Then either $T^{2}=0$ and $T^{+}$is a simple group, or $T$ is a simple ring. Results (1) and (2) follow immediately.

Note that in Proposition 3.3, if $R$ also satisfies a permutation identity then, in part (2), $\langle R, R\rangle \subseteq M$.

\section{References}

[1] V. Andrunakievic, 'Radicals in associative rings I', Mat. Sb. 44 (1958), 179-212.

[2] G. Birkenmeier and H. Heatherly, 'Polynomial identity properties for near-rings on certain groups', Near-Ring Newsletter 12 (1989), 5-15.

[3] __, 'Medial near-rings', Monatsh. Math. 107 (1989), 89-110.

[4] __ , 'Medial and distributively generated near-rings', Monatsh. Math. 109 (1990), 97101.

[5] __ 'Medial rings and an associated radical', Czechoslovak Math. J. 40 (1990), 258283.

[6] _ , 'Permutation identity rings and the medial radical', in: Proc. Conference on Non-Commutative Ring Theory (Athens, Ohio), Lecture Notes in Math. 1448 (Springer, Berlin, 1990), pp. 125-138.

[7] _ , 'Minimal ideals in near-rings', Comm. Algebra 20 (1992), 457-468.

[8] H. Brown, 'Near algebras', Illinois J. Math. 12 (1968), 215-227.

[9] __ 'Distributor theory in near-rings', Comm. Pure Math. 21 (1968), 535-544.

[10] J. R. Clay, 'Near-rings on groups of low order', Math. Z. 104 (1968), 364-371.

[11] N. Divinsky, Rings and Radicals (Univ. of Toronto Press, Ontario, 1965).

[12] A. Fröhlich, 'Distributively generated near-rings (I. Ideal theory)', Proc. London Math. Soc. 8 (1958), 76-94.

[13] H. Heatherly and S. Ligh, 'Pseudo-distributive near-rings', Bull. Austral. Math. Soc. 12 (1975), 449-456.

[14] K. Kaarli, 'Minimal ideals in near-rings', Tartu Riikl. Ül. Toimetised, 336 (1975), 105142 (in Russian).

[15] _ _ 'On Jacobson type radicals of near-rings', Acta Math. Hung. 50 (1987), 71-78.

[16] _ , 'On minimal ideals of distributively generated near-rings', preprint.

[17] J. D. P. Meldrum, Near-rings and their links with groups (Pitman, Marshfield, MA, 1985).

[18] M. Putcha and A. Yaqub, 'Semigroups satisfying permutation identities', Semigroup Forum 3 (1971), 68-73.

[19] S. D. Scott, 'Minimal ideals in near-rings with minimal condition', J. London Math. Soc. (2) 8 (1974), 8-12.

[20] R. Wiegandt, 'On subdirectly irreducible near-rings which are fields', in: Near-Rings and Near-Fields (ed. G. Betsch) (Amsterdam, North-Holland, 1987) pp. 295-298.

\section{University of Southwestern Louisiana}

Lafayette, Louisiana 70504

U.S.A. 